\title{
EchoGéo
}

28 | 2014

Police : les espaces de l'ordre, l'ordre en espace

\section{Notices bibliographiques}

Notices bibliographiques issues de la Bibliographie Géographique Internationale (base FRANCIS) pour les requêtes « insécurité », " délinquance ", « criminalité ", « sécurité ", " politique urbaine », " gestion urbaine ", " contrôle ", « ordre public ».

\section{(2) OpenEdition}

Journals

Édition électronique

URL : https://journals.openedition.org/echogeo/13855

DOI : 10.4000/echogeo.13855

ISSN : 1963-1197

Éditeur

Pôle de recherche pour l'organisation et la diffusion de l'information géographique (CNRS UMR 8586)

Référence électronique

« Notices bibliographiques », EchoGéo [En ligne], 28 | 2014, mis en ligne le 08 juillet 2014, consulté le 31 juillet 2021. URL : http://journals.openedition.org/echogeo/13855 ; DOI : https://doi.org/10.4000/ echogeo. 13855

Ce document a été généré automatiquement le 31 juillet 2021.

EchoGéo est mis à disposition selon les termes de la licence Creative Commons Attribution - Pas d'Utilisation Commerciale - Pas de Modification 4.0 International (CC BY-NC-ND) 


\section{Notices bibliographiques}

Notices bibliographiques issues de la Bibliographie Géographique Internationale (base FRANCIS) pour les requêtes « insécurité », " délinquance », " criminalité », « sécurité », " politique urbaine », « gestion urbaine ", « contrôle ", « ordre public ».

\section{NOTE DE L'ÉDITEUR}

Ces notices bibliographiques sont publiées avec l'autorisation de l'INIST.

1 Légende des zones des références bibliographiques : FT : Titre en français ; ET : Titre en anglais; ST : Titre en espagnol; GT: Titre en allemand; OT : Titre en italien; AU : Auteur; DT: Type de document (Thèse, Congrès); SO: Source; FA: Résumé en français ; EA : Résumé en anglais ; FD : Mots-clés français.

ET: "The softer side of security": the role of social development in Cape Town's policing network

$\mathrm{Au}$ : PAASCHE (T.F.)

DT : Publication en série; Niveau analytique

SO : Geoforum; ISSN 0016-7185; Coden GFRMAK; Royaume-Uni; Da. 2013; Vol. 45;Pp. 259-265; Bibl. 49 ref.; 1 fig.

FA: L'accent est mis sur les accords de gouvernance pratiqués par certaines organisations qui œuvrent dans le développement social au Cap. Les acteurs du développement social sont associés au versant le plus doux de la sécurité, et de tels programmes ont été mis en place dans des "Improvement Districts". Les offres de la sécurité privée et celles du développement social deviennent complémentaires. La stratégie de contrôle social et de lutte contre la pauvreté devient plus efficace.

FD : Afrique du Sud; Cape Town; Western Cape; Développement social; Contrôle social; Lutte contre la pauvreté; Sécurité; Organisation; Politique urbaine; Gestion urbaine; Sans-abri

ET: Immigrants, banlieues, and dangerous things: ideology as an aesthetic affair

AU : DIKEÇ (M.)

DT : Publication en série; Niveau analytique 
SO : Antipode (Oxford. Print); ISSN 0066-4812; Royaume-Uni; Da. 2013; Vol.45; No. 1; Pp. 23-42; Bibl. 3 p.

FA : L'auteur analyse la notion d'idéologie en réunissant la théorie d'Ernesto Laclau de la spécificité de l'idéologie et la notion de Jacques Rancière des régimes esthétiques. L'exemple de la politique sécuritaire des précédents gouvernements français illustre la mise en place des preuves perceptibles fournissant les conditions légitimes et la normalisation de ces pratiques. Il conclut que les conséquences les plus perverses de cette idéologie sont le renforcement de ces régimes, facilitant ainsi l'idée de consensus autour d'eux.

FD : France; Géographie politique; Concept; Idéologie; Rôle de l'État; Banlieue; Esthétique; Théorie; Sécurité; Immigrés; Police; Politique gouvernementale

\section{FT : Les villes face à l'insécurité}

DT : Publication en série; Niveau monographique

SO : Cahiers de l'Institut d'aménagement et d'urbanisme de la région d'Ile-de-France; ISSN 0153-6184; France; Da. 2010; No. 155; 88 p.

FA : Nécessité de mener des enquêtes portant sur la victimation et le sentiment d'insécurité des Franciliens. Des travaux plus opérationnels sont également développés. Il s'agit d'analyser les phénomènes de délinquance et les politiques mises en œuvre pour y répondre. Ces approches participent à une coproduction de sécurité, associant des domaines divers. Dans ce fascicule, la parole est donnée à des acteurs, à des chercheurs et à des experts de ce domaine complexe et sensible.

FD : Ville; Insécurité; France; Ile-de-France; Délinquance; Société urbaine; Sentiment de peur; Gestion urbaine; Politique sociale; Police; Politique urbaine

5 FT : Les Missions de Police routière de la Sûreté du Québec : entre politique publique, logique territoriale et contraintes économiques

$\mathrm{AU}$ : CARNIS (L.)

DT : Publication en série; Niveau analytique

SO : Revue d'économie régionale et urbaine; ISSN 0180-7307; France; Da. 2011; No. 4; Pp. 765-787; Bibl. 2 p.; 1 fig.

FA: La mise en œuvre d'une politique publique de sécurité routière nécessite l'activation de certains relais, dont l'organisation policière. Les agences policières sont en charge de missions différentes. Elles constituent un mandat que leur a confié l'autorité politique, et elles peuvent être redéfinies compte tenu de l'évolution de l'environnement. Elles dépendent aussi de contraintes organisationnelles internes. D'où la mobilisation du modèle de Porter pour traduire l'évolution des missions de police routière de la Sûreté du Québec

FD : Police; Sécurité routière; Organisation; Canada; Québec; Stratégie d'acteurs; Institution; Politique

6 FT : La production de la sécurité par l'Etat. Une illustration par la sécurisation de la mobilité transfrontalière dans l'Union européenne et ses espaces du risque : Géographie de la sécurité et de l'insécurité)

GT: Wie und warum Staat Sicherheit produziert. Dargestellt anhand der Versicherheitlichung grenzüberschreitender Mobilität seitens der EU und der so produzierten Räume des Risikos : Geographie der (Un-) Sicherheit

AU : BELINA (B.); KORF (B.); OSSENBRÜGGE (J.)

DT : Publication en série; Niveau analytique

SO : Geographica helvetica; ISSN 0016-7312; Coden GGHVA4; Suisse; Da. 2010;No. 3; Pp. 
189-197; Bibl. 3 p.; 1 fig.

FA : L'auteur traite de l'approche de la gouvernementalité de Foucault en l'inscrivant dans les théories matérialistes de l'État. Il contextualise cette problématique à l'aide des concepts de risque et de sécurisation. Il illustre les techniques de gouvernementalité du risque et de la sécurisation en faisant référence aux récentes politiques de l'Union européenne en matière de mobilité transfrontalière et de migration, en insistant particulièrement sur les « espaces du risque » produits par ce contexte.

FD : Europe; Union européenne; Migration internationale; Politique de l'immigration; Risque; Gouvernementalité; Frontière; Production de l'espace; Territoire; État; Sécurité

7 FT : (Géographies de la sécurité et de l'insécurité)

GT : Geographie der (Un-) Sicherheit

AU : KORF (B.); OSSENBRÜGGE (J.)

DT : Publication en série; Niveau monographique

SO : Geographica helvetica; ISSN 0016-7312; Coden GGHVA4; Suisse; Da. 2010;No. 3; Pp. 167-228.

FA : Les auteurs abordent le thème de la sécurité et de l'insécurité au travers des articles suivants :1) le concept de "ungoverned territories" et la politique de sécurité aux États-Unis ; 2) la production de la sécurité par l'État. Une illustration par la sécurisation de la mobilité transfrontalière dans l'Union Européenne et ses espaces du risque ; 3) le changement climatique comme problème de sécurité ; 4) l'(in) sécurité fluide et la spatialité des commerces de rue brésiliens ; 5) les banlieues comme contrelieux de la République et les nouvelles politiques de sécurité dans les périphéries françaises.

FD : États-Unis; Union européenne; Brésil; France; Espace urbain; Sécurité; Territoire; Terrorisme; Souveraineté; Gouvernementalité; Risque; Climat; Changement climatique; Commerçant; Développement urbain; Commerce de détail; Rue; Banlieue; Identité nationale; Police; État; Europe; Frontière

ET: Crime management and urban governance : everyday interconnections in South Africa

AU : METH (P.)

DT : Publication en série; Niveau analytique

SO : Environment \& planning A (Print); ISSN 0308-518X; Royaume-Uni; Da. 2011; Vol. 43; No. 3; Pp. 742-760; Bibl. 28 ref.; 6 fig.

EA: The paper uses a case study from South Africa to understand the micro local experiences of the interconnections between what is described as "crime management" and local governance. The extent of interconnection is beyond that captured by the concept of "partnership", as multiple governance structures engage in crime management. The interconnection is theorised as the criminalisation of governance within a context of state-building.

FD : Afrique du Sud; Criminalité; Gestion urbaine; Politique locale; Politique sociale; Politique urbaine

ET: Spatial methodologies for studying crime

AU : LEBEAU (J.L.); LEITNER (M.)

DT : Publication en série; Niveau analytique

SO : The Professional geographer; ISSN 0033-0124; Etats-Unis; Da. 2011; Vol.63; No. 2; Pp. 161-261. 
FA : Les auteurs proposent différentes méthodologies spatiales appliquées à l'étude du crime aux États-Unis , en particulier dans les villes de Baltimore, Chicago, Philadelphie, Camden et La Nouvelle-Orléans. Les articles proposés traitent successivement de l'effet des caractéristiques du quartier sur la délinquance et la récidive des jeunes, de la population ambiante et l'analyse du crime, d'une méthodologie de profilage géographique, de la diffusion spatiale des crimes et la mesure des effets des opérations de police et pour terminer, de l'analyse spatiale et temporelle de l'impact de l'ouragan Katrina sur le signalement des crimes en Louisian.e

FD : États-Unis; Géographie sociale; Jeunes; Justice; Criminalité; Localisation; Analyse spatiale; Ville; Police; Quartier

10 ET: Exclusionary policies are not just about the "neoliberal city": acritique of theories of urban revanchism and the case of Rotterdam

AU : VAN EIJK (G.)

DT : Publication en série; Niveau analytique

SO : International journal of urban and regional research; ISSN 0309-1317; RoyaumeUni; Da. 2010; Vol. 34; No. 4; Pp. 820-834; Bibl. 2 p.; 1 tabl.

FA: Ces politiques d'exclusion sont interprétées comme un "revanchisme urbain" : il s'agit de séduire touristes et acteurs de la gentrification, aux dépens de groupes marginaux ou minoritaires. L'auteur se demande si elles sont motivées par les insécurités économiques et la volonté d'attirer le capital. Ces politiques sont liées aux idées sur le multiculturalisme et l'intégration. Les préoccupations d'unité nationale jouent aussi un rôle, la création de quartiers mixtes constituant une stratégie d'inclusion excluant dans les faits des individus. Les théories du revanchisme doivent incorporer une notion plus complexe de la "sécurité" et de la façon dont les insécurités produisent des stratégies d'exclusion et d'inclusion.

FD : Pays-Bas; Rotterdam; Exclusion sociale; Politique urbaine; Embourgeoisement; Quartier; Ordre social; Citoyenneté; Sécurité

11 ET: Security in public place: an empirical assessment of three US cities

AU : NÉMETH (J.)

DT : Publication en série; Niveau analytique

SO : Environment \& planning A (Print); ISSN 0308-518X; Royaume-Uni; Da.2010; Vol. 42; No. 10; Pp. 2487-2507; Bibl. 44 ref.; 7 fig., 8 tabl.

FA : L'auteur donne des indications sur la fréquentation des Centres Civiques et des Districts Financiers à New York City, Los Angeles et San Francisco. Dans chaque quartier sont décrits les paysages sécuritaires grâce à une évaluation de l'intensité, de la durée et de la localisation des zones de sécurité individuelle. Ce paysage couvre de $3,4 \%$ à $36 \%$ de l'espace accessible au public, l'intensité atteignant des niveaux supérieurs à New York City. La gestion par des acteurs multiples est un gage de plus grande sécurité que la gestion par une entité unique.

FD : Sécurité; Espace public; États-Unis; Espace urbain; Politique urbaine; Localité; Quartier; New York City; Los Angeles; San Francisco

ET: Producing spaces for representation: racist marches, counterdemonstrations, and public-order policing

AU : WAHLSTRÖM (M.)

DT : Publication en série; Niveau analytique

SO : Environment and planning. D. Society \& space; ISSN 0263-7758; Royaume-Uni; Da. 2010; Vol. 28; No. 5; Pp. 811-827; Bibl. 39 ref.; 2 tabl. 
FA : L'auteur étudie comment l'espace est produit à travers l'interaction entreles démonstrations et protestations politiques au cours d'actions racistes et antiracistes en Suède. Proposition d'un modèle pour une analyse spatiale de la police et des protestataires basée sur les concepts de territorialisation et de déterriorialisation.

FD : Suède; Production de l'espace; Contrôle territorial; Activisme; Police; Territoire; Racisme; Symbolique de l'espace; Politique

FT : Partager la fonction répressive : la collaboration entre la police et une compagnie de sécurité privée dans un quartier aisé du Cap : Puissance émergente, nation adolescente : l'Afrique du Sud en 2010

AU : ROUMET (R.)

DT : Publication en série; Niveau analytique

SO : EchoGéo; ISSN 1963-1197; France; Da. 2010; No. 13; Pp. 1-11; Bibl. 15 ref.; 1 fig. coul.

FA : Basé sur une étude qualitative menée dans une banlieue aisée du Cap, cet article examine les mutations de la fonction policière induites par le recrutement d'une compagnie de sécurité privée pour assurer l'ordre public et la sécurité. Libérés des patrouilles assurées par la compagnie de sécurité, les policiers voient leur action recentrée sur le développement de services administratifs et de campagnes de prévention. La police locale et une entreprise privée collaborent pour former, avec la participation active des habitants, un exemple atypique de recomposition de la gouvernance sécuritaire en Afrique du Sud.

FD : Banlieue; Afrique du Sud; Western Cape; Cape Town; Sécurité; Gouvernance; Police; Prévention; Géographie urbaine; Ordre public; Participation

ET: Ungoverned space: global security and the geopolitics of broken windows

AU : MITCHELL (K.); POWER (M.); CAMPBELL (D.)

DT : Publication en série; Niveau analytique

SO : Political geography; ISSN 0962-6298; Royaume-Uni; Da. 2010; Vol. 29; No. 5; Pp. 289-297; Bibl. 129

FA : Les lieux abandonnés sans surveillance, vécus comme dangereux et peu sûrs, font l'objet de toutes les attentions de la part de l'administration américaine au moyen de politiques sécuritaires et de gestion spatiale. L'auteur illustre son propos au travers de l'exemple de la ville de New York et du vocabulaire employé dans le cadre de la politique de surveillance (celle des vitres cassées) et de tolérance zéro. On assiste à la mise en place d'une géopolitique des lieux sans surveillance, désordonnés, produisant une nouvelle forme de gouvernance issue de la sécurité qui a été aussi appliquée en Irak.

FD : États-Unis; New York State; New York City; Géopolitique; Espace urbain; Sécurité; Gouvernance; Criminalité; Police; Cartographie automatique; Iraq; Insécurité; Ville

\section{ET: Transecting security and space in Maputo}

AU : PAASCHE (T.F.); SIDAWAY (J.D.)

DT : Publication en série; Niveau analytique

SO : Environment \& planning A (Print); ISSN 0308-518X; Royaume-Uni; Da. 2010; Vol. 42; No. 7; Pp. 1555-1576; Bibl. 85; 1 fig., 9 phot.

FA : Au cours de déplacements dans le centre de Maputo, les auteurs étudient la prise en main par des sociétés privées de la sécurité des lieux et des habitations. Ils mettent en évidence la spatialisation et la fragmentation de la sécurité dans une ville où l'Etat est incapable d'assurer ses missions régaliennes. Le second objectif de cet article est l'approche pédestre de l'environnement urbain, basée sur la psychogéographie des 
lieux.

FD : Mozambique; Maputo; Ville; Centre-ville; Espace urbain; Post-colonialisme; Sécurité; Psycho-géographie; Police; Privatisation; Trajet; Perception

ET: Security zones and New York City's shrinking public space

AU : NÉMETH (J.); HOLLANDER (J.)

DT : Publication en série; Niveau analytique

SO : International journal of urban and regional research; ISSN 0309-1317; RoyaumeUni; Da. 2010; Vol. 34; No. 1; Pp. 20-34; Bibl. 44 ref.; 5 fig., 2 tabl., 5 phot.

FA : Des propriétaires et gestionnaires de bâtiments publics, banques et tribunaux ont fermé des rues et équipé l'espace environnant d'obstacles en béton, de plots et de quasi-fossés afin de parer aux attaques terroristes potentielles. Ces protections sont normales en situations d'urgence, mais lorsque la menace décroît, les zones concernées ne parviennent pas à diversifier leurs usagers, l'espace étant réservé aux détenteurs de droits d'accès. Une enquête dans deux quartiers éminents de New York montre que plus d'un quart de la surface cumulée non bâtie est désormais dans une zone sécurisée. Cette proportion est similaire, donnant un éclairage sur la façon dont les cibles terroristes sont définies et justifiées sur les plans intérieur et extérieur.

FD : Sécurité; Espace public; Ordre public; Espace urbain; Vie sociale; Démocratie; Lutte contre le terrorisme; États-Unis; New York State; New York City

FT : Embellissement sous surveillance: une géographie des politiques de réaménagement des espaces publics au centre de Bruxelles

AU : DESSOUROUX (C.); VAN CRIEKINGEN (M.); DECROLY (J.-M.)

DT : Publication en série; Niveau analytique

SO : Belgeo (Leuven); ISSN 1377-2368; Belgique; Da. 2009; No. 2; Pp. 169-186; Bibl. 35 ref.; 3 fig., 4 phot.

FA : Localisations des interventions sur les espaces publics de la ville centrale. Pour les quartiers populaires notamment, on met en évidence un assemblage d'interventions portant sur l'esthétique urbaine et des actions qui visent à normaliser les usages de l'espace urbain central, que l'on peut qualifier « d'embellissement sous surveillance». Dispositifs qui cherchent moins à isoler des espaces de relégation sociale du reste du territoire qu'à promouvoir le réinvestissement des quartiers populaires centraux par les classes moyennes (idéal de mixité sociale). Schéma qui s'inscrit dans un glissement plus large vers un modèle « d'État social-sécuritaire ».

FD : Espace public; Régénération urbaine; Sécurité; Belgique; Bruxelles; Espace urbain; Aménagement urbain; Politique urbaine; Centre-ville; Esthétique du paysage

FT : Le défi des "teichopolitiques". Analyser la fermeture contemporaine des territoires

AU : BALLIF (F.); ROSIÈRE (S.)

DT : Publication en série; Niveau analytique

SO : Espace géographique (Paris); ISSN 0046-2497; Coden ESGEDD; France; Da. 2009; Vol. 38; No. 3; Pp. 193-206; Bibl. 33 ref.; 4 fig., 2 phot.

FA: On imagine souvent que la mondialisation implique la disparition des murs. Pourtant, de nouveaux systèmes de fermeture sont édifiés aussi bien aux frontières que dans les villes. Les auteurs analysent des "teichopolitiques" (néologisme construit sur une racine grecque, "mur" de la cité), fondées sur la fermeture des territoires. L'enjeu sécuritaire est plus ou moins sensible, mais la dimension économique aussi: la construction de barrières est un marché rentable. Entreprises et promoteurs jouent sur 
les peurs dominantes et leurs produits, recherchés par les gouvernements, les édiles ou les citadins, contribuent à la prolifération des barrières.

FD : Mondialisation; Frontière; Communauté fermée; Effet de barrière; Enfermement; Sécurité; Contrôle spatial; Ville

ET: In the service of tyranny : debating the role of planning in Zimbabwe's urban "clean-up" operation

AU : KAMETE (A.Y.)

DT : Publication en série; Niveau analytique

SO : Urban studies (Harlow); ISSN 0042-0980; Royaume-Uni; Da. 2009; Vol. 46;No. 4; Pp. 897-922; Bibl. 4 p.; 2 tabl.

FA : Rôle de la planification dans l'Opération controversée de 2005 "Restaurer l'ordre", campagne de "nettoyage" urbain. Examen critique de deux points de vue relatifs à la planification menée par un régime qui ne recueille guère d'approbation internationale. Relations entre les actions des planificateurs et la répression menée par l'État au cours de l'opération.

FD : Planification; Stratégie d'acteurs; Contrôle social; Rôle de l'Etat; Sécurité; Politique urbaine; Ordre public; Structure socio-économique; Zimbabwe; Développement urbain

FT : La fermeture municipale de rues publiques à Londres : le cas des Gating Orders à Camden

AU : LAKEHAL (L.)

DT : Publication en série; Niveau analytique

SO : Géocarrefour (Lyon); ISSN 1627-4873; France; Da. 2008; Vol. 83; No. 2;Pp. 129-139; Bibl. 28 ref.; 3 tabl., 2 cartes.

FA : Les politiques urbaines mises en œuvre dans chaque borough sont influencées par le principe de prévention de la criminalité par l'urbanisme. L'auteur analyse la procédure inédite du " décret de fermeture » mise en œuvre dans un borough pionnier en matière d'urbanisme "situationnel ». Fermeture municipale de certaines rues publiques jugées criminogènes. Hypothèse d'une certaine banalisation de la sécurisation et de la fermeture d'espaces publics en Angleterre.

FD : Espace urbain; Espace public; Urbanisme; Privatisation; Insécurité; Politique urbaine; Délinquance; Royaume-Uni; England; London

21 FT : (Relations locales dans un township post-apartheid d'Afrique du Sud)

OT : Mens-plaats relaties in een Zuid-Afrikaanse post-apartheid township

AU : MEYS (S.)

DT : Publication en série; Niveau analytique

SO : De Aardrijkskunde ; Belgique; Da. 2007; No. 2-3; Pp. 63-73; Abs. anglais; Bibl. 1 p.; 4 fig., 7 phot., 4 cartes.

FA: Même dans un monde de globalisation, les expériences personnelles et les interactions sont vécues à l'échelle locale et ont des impacts considérables sur les activités et le sens de la hiérarchie au niveau du voisinage. Une étude réalisée dans le quartier de Westbank dans la ville du Cap montre que, aussi longtemps que la situation ne sera pas stabilisée et sécurisée, le quartier manquera d'opportunités pour que les résidents bénéficient de meilleures conditions de vie.).

FD : Post-apartheid; Géographie urbaine; Police privée; Quartier; Sécurité; Recherche participative; Conditions de vie; Afrique du Sud; Western Cape; Cape Town

ET: Mumbai's development mafias: globalization, organized crime and land development 
AU : WEINSTEIN (L.)

DT : Publication en série; Niveau analytique

SO : International journal of urban and regional research; ISSN 0309-1317;

Royaume-Uni; Da. 2008; Vol. 32; No. 1; Pp. 22-39; Abs. anglais; Bibl. 2 p.

FA : L'article étudie comment les réformes politiques des quinze dernières années en Inde ont reconfiguré les marchés immobiliers et les politiques d'aménagement foncier. Les syndicats locaux du crime ont saisi les occasions politiques créées par ces changements pour gagner en influence sur l'aménagement foncier. Depuis le début des années 1990, libéralisation et déréglementation ont réduit la demande pour les biens de consommation de contrebande, poussant ces syndicats à diversifier leurs opérations, d'où leur investissement dans la promotion immobilière et leur rôle central dans les politiques d'urbanisme.

FD : Gestion urbaine; Marché immobilier; Criminalité; Organisation; Foncier; Réseau; Secteur informel; Urbanisme; Inde; Maharashtra; Bombay

ET: Civil liberties and the regulation of public space: the case ofsidewalks in Las Vegas

AU : BLUMENBERG (E.); EHRENFEUCHT (R.)

DT : Publication en série; Niveau analytique

SO : Environment and planning. A; ISSN 0308-518X; Royaume-Uni; Da. 2008;Vol. 40; No. 2; Pp. 303-322; Abs. anglais; Bibl. 53 ref.; 1 fig., 1 tabl.

FA : L'accent est mis sur la réglementation de l'espace public et sur les droits qui lui sont associés. Les auteurs étudient comment les gestionnaires locaux ont fait le lien entre des usages variés et concurrentiels des trottoirs. Importants enjeux financiers.

FD : Espace urbain; Espace public; Gestion urbaine; Trottoir; Environnement urbain; Piétons; Privatisation; Sécurité; Développement urbain; États-Unis; Nevada; Las Vegas

\section{ET: Badlands of the Republic : space, politics and urban policy}

AU : DIKEÇ (M.)

DT : Livre; Niveau monographique

SO : Badlands of the Republic : space, politics and urban policy; Royaume-Uni; Oxford: Blackwell; Da. 2007; Pp. 220 p.; ISBN 978-1-4051-5630-1

EA : This monograph provides a comprehensive account of urban policy in France whilst incorporating reflections on space and politics. It also examines the politics of immigration and the contemporary transformations of the state in France. The author challenges overarching generalizations that tend, in the anglo-saxon literature, to be based almost exclusively on the British and North American experiences. It focuses on the revolts in the "banlieues" of French cities during 2005 and covers related topics such as those associated with citizenship, identity and immigration. Attention is drawn to the role of the police in the suburbs, the quest for social justice, and labelling of "dangerous neighbourhoods". Particular stress is placed on the example of Vaulx-enVelin.

FD : Politique urbaine; Politique de l'immigration; Banlieue; Identité; Sécurité; Quartier; Ordre public; France

ET: The emergence of a "City of Cages" in Lima: neighbourhood appropriation in the context of rising insecurities

AU : PLÖGER (J.)

DT : Publication en série; Niveau analytique

SO : Cybergeo; ISSN 1278-3366; France; Da. 2007-06-05; No. 377; 16 p.; Abs.anglais/ 
français; Bibl. 41 ref.; 2 cartes, 3 phot.

FA : La plupart de ces enclaves sont en fait des quartiers qui ont été enclos. Elles sont présentes dans des contextes socio-économiques variés. Caractère hautement informel de ces installations. Facteurs qui déterminent l'implantation, interactions avec les autres services de sécurité et importance de l'organisation résidentielle. Contexte des transformations liées à la récession des années 1980 et aux ajustements structurels des années 1990. L'État semble incapable de satisfaire la demande de services publics, et les acteurs prolifèrent afin de pallier ces carences.

FD : Communauté fermée; Résidence; Quartier; Structure socio-économique; Insécurité; Restructuration économique; Services de sécurité; Contrôle social; Espace urbain; Pérou; Lima

ET: Mapping the labyrinth from within : the political economy of Nicaraguan youth policy concerning violence

AU : ROCHA GÓMEZ (J.L.)

DT : Publication en série; Niveau analytique

SO : Bulletin of Latin American Research; ISSN 0261-3050; Royaume-Uni; Da.2007; Vol. 26; No. 4; Pp. 533-549; Abs. anglais; Bibl. 47 ref.

FA: Approche méthodologique dans l'étude de deux organisations gouvernementales nicaraguayennes qui sont concernées par le phénomène de la violence de la jeunesse : la Police Nationale et l'office de l'Ombudsman pour les Enfants et les Adolescents. L'auteur met en évidence leur fonctionnement et leur dysfonctionnement ainsi que leurs actions et leurs pratiques qui ne sont pas systématiquement coordonnées et parfois divergentes pour tenter de juguler la délinquance, la violence, voire la criminalité des jeunes à défaut de pouvoir la prévenir.

FD : Législation; Institution; Politique; Politique sociale; Violence; Délinquance; Criminalité; Gang; Prévention; Vulnérabilité; Jeunes; Population locale; Perception; Espace vécu; Police; Ordre public; Justice sociale; Nicaragua

ET: The geographies of policing

AU : YARWOOD (R.)

DT : Publication en série; Niveau analytique

SO : Progress in human geography; ISSN 0309-1325; Royaume-Uni; Da. 2007;Vol. 31; No. 4; Pp. 447-465; Abs. anglais; Bibl. 4 p.; 1 tabl.

EA: This article reviews geographical progress in this area and argues that attention should be shifted from the police towards policing. Consideration is given to the increasing numbers of agencies that perform policing, including state, private and voluntary actors. The concept of governance provides a suitable framework for theorizing new geographies of policing.

FD : Police; Gouvernance; Géographie; Néolibéralisme; Criminalité; Insécurité; Concept

ET: Governmentality, calculation, territory

AU : ELDEN (S.)

DT : Publication en série; Niveau analytique

SO : Environment and planning. D : Society and space; ISSN 0263-7758; Royaume-Uni; Da. 2007; Vol. 25; No. 3; Pp. 562-580; Abs. anglais; Bibl. 62 ref.

FA : Débat à propos de deux cours de Foucault récemment publiés : "Sécurité, territoire, population" et "Naissance de la biopolitique".

FD : Gouvernementalité; Territoire; Sécurité; Foucault (M.); Planification urbaine; Stratégie territoriale; Pouvoir; État; Politique 


\section{AU : BENTON-SHORT (L.)}

DT : Publication en série; Niveau analytique

SO : Environment and planning. D : Society and space; ISSN 0263-7758; Royaume-Uni; Da. 2007; Vol. 25; No. 3; Pp. 424-446; Abs. anglais; Bibl. 77 ref.; 2 fig., 4 phot.

FA : Étude du paradoxe de la démocratie et de l'hypersécurité dans l'un des espaces publics les plus symboliques de Washington. Examen du Plan de sécurité de 2002. Un débat public animé a été engagé à cette occasion.

FD : Sécurité; Lutte contre le terrorisme; Symbolique de l'espace; Espace urbain; Espace public; Planification urbaine; Politique urbaine; États-Unis; Washington D.C.; Washington

\section{l'implantation des ensembles résidentiels sécurisés dans la capitale} mozambicaine

$\mathrm{AU}$ : FOLIO (F.)

DT : Publication en série; Niveau analytique

SO : Annales de géographie (Paris); ISSN 0003-4010; France; Da. 2007; Vol. 116; No. 655; Pp. 247-270; Abs. français/anglais; Bibl. 31 ref.; 2 fig., 2 phot.

FA : L'implantation de ces complexes résidentiels est révélatrice d'un courant néolibéral. L'ouverture récente aux programmes de coopération et aux investissements exogènes a vu immigrer une population allochtone en quête de logements viables et sécurisés. Il paraît difficile d'écarter le motif sécuritaire quant à ce choix de vie, bien qu'il ne soit pas le seul. Cette émergence opère dans un cadre foncier assez opaque, dont tire profit une quantité non négligeable d'acteurs spécifiques. Cela conduit à une certaine homogénéisation de ce quartier littoral.

FD : Criminalité; Ségrégation; Insécurité; Communauté fermée; Fragmentation urbaine; Logement; Spéculation foncière; Politique urbaine; Promotion immobilière; Mozambique; Maputo

$31 \quad$ FT : La gestion d'un territoire hétérogène : le cas des missions de police routière de la Traffic Branch du New South Wales Police Service

AU : CARNIS (L.)

DT : Publication en série; Niveau analytique

SO : Revue d'économie régionale et urbaine; ISSN 0180-7307; France; Da. 2006; No. 1; Pp. 131-157; Abs. français/anglais; Bibl. 59 ref.; 8 fig.

FA : Les outils de l'analyse économique sont appliqués au cas particulier de la police routière de Nouvelle-Galles-du-Sud. L'auteur souligne la gestion hétérogène d'une juridiction et des modalités d'ajustement dont dispose l'organisation, dans lesquelles un processus de rationalisation économique semble être à l'œuvre. L'approche théorique permet de cerner les conséquences attendues d'une hétérogénéité de la zone d'intervention pour l'allocation des ressources.

FD : Organisation; Police; Théorie économique; Allocation; Modèle; Sécurité routière; Investissement; Australie; New South Wales

ET: Two decades of French urban policy : from social development of neighbourhoods to the republican penal state

AU : DIKEÇ (M.)

DT : Publication en série; Niveau analytique

SO : Antipode (Oxford); ISSN 0066-4812; Royaume-Uni; Da. 2006; Vol. 38; No.1; Pp. 59-81;

EchoGéo, 28 | 2014 
Abs. anglais; Bibl. 60 ref.

FA: La restructuration de l'État français diffère sensiblement du néolibéralisme américain ou anglais, en partie à cause de la tradition républicaine qui renforce le rôle de l'État pour le bien-être de ses citoyens. Cette restructuration porte les signes d'une forte tradition de l'État français et peut être vue comme une articulation du néolibéralisme avec des traditions politiques.

FD : Politique urbaine; Néolibéralisme; Rôle de l'État; Banlieue; Efficacité économique; Protection sociale; Politique; Développement social; Logement social; Sécurité; France

\section{FT : La gendarmerie, force de sécurité au service de la nation}

AU : CHICHIGNOUD (C.)

DT : Publication en série; Niveau analytique

SO : Hérodote (Paris); ISSN 0338-487X; France; Da. 2005; No. 116; Pp. 82-94; Abs. français/anglais; Bibl. 13 ref.

FA : La gendarmerie, bien que force militaire, exerce des missions surtout civiles et de service public. Cette spécificité pose problème dès qu'est abordée la question des liens avec la nation : cela peut être prétexte à une remise en cause de l'appartenance de la gendarmerie au monde des armées. Derrière ces discours se trouvent des rivalités de pouvoir parfois historiques entre la gendarmerie et les autres composantes de l'armée. La référence à la nation est saisie différemment selon la place dans la hiérarchie, les responsabilités exercées. Le discours sur la nation est plus ou moins présent suivant les métiers de gendarme et les perspectives de carrière.

FD : Gendarmerie; Nation; Armée; Discours; Sécurité; Citoyenneté; Service public; Profession; Secteur public; France

ET: Investigating the spatiotemporal links between disorder, crime, and the fear of crime

AU : DORAN (B.J.); LEES (B.G.)

DT : Publication en série; Niveau analytique

SO : The Professional geographer; ISSN 0033-0124; Etats-Unis; Da. 2005; Vol.57; No. 1; Pp. 1-12; Abs. anglais; Bibl. 41 ref.; 4 fig., 1 tabl., 2 phot.

FA : Un SIG permet d'explorer les liens spatio-temporels potentiels entre ces processus à Wollongong, Nouvelle-Galles-du-Sud. L'accent est mis sur les rapports entre les graffitis et la peur du crime. La distribution de la peur du crime varie beaucoup au cours du temps et coïncide spatialement avec les concentrations du désordre. Les graffitis sont l'un des types les plus prévalents du désordre physique. Les résultats sont discutés dans le cadre de la théorie du "bris de vitres" et de l'intervention stratégique à l'échelle de la communauté.

FD : Criminalité; Comportement; Recherche; Système d'information géographique; Ordre public; Espace-temps; Attitude; Analyse spatiale; Peur du crime; Australie; New South Wales; Wollongong

ET: Crime and disorder, and house sales and prices around the casino sites in Windsor, Ontario, Canada

AU : PHIPPS (A.G.)

DT : Publication en série; Niveau analytique

SO : Canadian geographer; ISSN 0008-3658; Canada; Da. 2004; Vol. 48; No. 4; Pp. 403-432; Abs. anglais/français; Bibl. 76 ref.; 6 fig., 10 tabl.

FA : Impact de l'ouverture et de la fermeture d'un nouveau casino urbain sur le crime, le désordre public, la vente et les prix des maisons dans le voisinage. Mesurés dans le 
temps, les chiffres hebdomadaires des infractions et les moyennes mensuelles du prix des maisons oscillent presque au hasard tout en restant proches de leurs moyennes, sans que l'ouverture ou la fermeture d'un nouveau casino les influence. Il est cependant prématuré de conclure que les casinos n'ont aucun effet sur les infractions et le prix des maisons

FD : Impact; Criminalité; Ordre public; Marché du logement; Coût du logement; Casino; Modèle; Espace-temps; Quartier; Espace urbain; Canada; Ontario; Windsor

FT : Conception et réalisation d'un système d'aide à la décision pour l'intervention opérationnelle en milieu urbain

AU : KESRAOUI (A.); OULD ALI (T.); TAKKA (H.)

DT : Publication en série; Niveau analytique

SO : Bulletin des sciences géographiques; ISSN 1112-3745; Algérie; Da. 2004; No. 14; Pp. 19-23; Abs. arabe/français/anglais; Bibl. 6 ref.; 7 fig.

FA : Les auteurs présentent leur méthodologie: mise en place d'une base de données urbaines (B.D.U.), dédiée à l'intervention opérationnelle, sous le logiciel S.I.G MapInfo. La base de données est de type relationnel, conçue selon la méthode MERISE. La 2ème étape est la conception d'une logique de processus décisionnel sous la forme d'un Système Interactif d'Aide à la Décision S.I.A.D. La dernière étape est l'intégration du S.I.G et du S.I.A.D afin d'avoir un S.I.A.D.R.S, (un Système Interactif d'Aide à la Décision à Référence Spatiale), destiné à la gestion des interventions des services de sécurité en milieu urbain.

FD : Espace urbain; Système d'information géographique; Aide à la décision; Sécurité; Gestion urbaine; Base de données; Méthodologie; Cartographie; Topologie

\section{RÉSUMÉS}

Liste des notices bibliographiques obtenues en interrogeant la Bibliographie Géographique Internationale (BGI - bgi-prodig.inist.fr) qui correspond au domaine Géographie de la base FRANCIS (INIST-CNRS, http://www.inist.fr). Pour cette rubrique l'interrogation a été faite sur « insécurité ", « délinquance », « criminalité », " sécurité », «politique urbaine », " gestion urbaine », « contrôle », « ordre public ». 